\title{
Ansiedad escénica y ejecución musical en estudiantes del conservatorio de música en Arequipa, 2020
}

\author{
Scenic Anxiety and Musical Performance in Students \\ of the Music Conservatory in Arequipa, 2020
}

José Luis Arias Gonzáles

Milagros del Rosario Cáceres Chávez

DOI 10.15517/es.v81i1.47276

(c) (i) () Esta obra está bajo una licencia Creative Commons
By NG ND Reconocimiento-No comercial-Sin Obra Derivada 


\title{
Ansiedad escénica y ejecución musical en estudiantes del conservatorio de música en Arequipa, 2020
}

\author{
Scenic Anxiety and Musical Performance in Students \\ of the Music Conservatory in Arequipa, 2020
}

\author{
José Luis Arias Gonzáles ${ }^{1}$ \\ Enfoques consulting \\ Arequipa, Perú \\ Milagros del Rosario Cáceres Chávez ${ }^{2}$ \\ Sinfonía por el Perú \\ Arequipa, Perú
}

Recibido: 11 de noviembre de 2020 Aprobado: 28 de abril de 2021

\begin{abstract}
Resumen
El objetivo general del estudio fue evaluar la relación entre la ansiedad escénica y la ejecución musical de los estudiantes de la Especialidad de Cuerdas Frotadas del Conservatorio Regional de Música "Luis Duncker Lavalle". Se realizó un estudio básico, con alcance correlacional y diseño no experimental-transversal. La muestra se conformó por 48 estudiantes de cuerdas frotadas y se utilizó el método no probabilístico, por conveniencia. Como técnicas se utilizaron el test y la observación, y como instrumentos se utilizaron el test del pingüino y la rúbrica de evaluación. Los resultados demostraron que el $71 \%$ de estudiantes tuvo un nivel de ansiedad alto y el $77 \%$ tuvo un nivel de ejecución musical deficiente. La prueba de hipótesis de Rho de Spearman estableció, con un valor de 0.000 ,

1 Investigador independiente en Enfoques Consulting, Perú. Maestro en Gerencia Social y Recursos Humanos por la Universidad Católica de Santa María. ORCID: 0000-0002-3250-5287. Correo electrónico: joseariasgon6@gmail.com

2 Instructora de violín en Sinfonía por el Perú. Maestra en Educación Superior por la Universidad Católica de Santa María. ORCID: 0000-0001-9091-5842. Correo electrónico: milagros_c1@hotmail.com
\end{abstract}


Ansiedad escénica y ejecución musical en estudiantes del

Artículo conservatorio de música en Arequipa, 2020

que existió una relación significativa entre las variables de estudio; mientras que el coeficiente de correlación de -.707 indicó que la relación fue inversa.

Palabras clave: música; artes escénicas; interpretación musical; ansiedad escénica; música clásica

\begin{abstract}
The general objective of the study was to evaluate the relationship between stage anxiety and musical performance of the students of the Strings Specialty at "Luis Duncker Lavalle" Regional Music Conservatory. A basic study was carried out with correlational scope and non-experimental cross-sectional design. The sample was made up of 48 string students and the non-probabilistic method was used - for convenience. As techniques the test and the observation were used and as instruments the penguin test and the evaluation rubric. The results showed that $71 \%$ of students had a high level of anxiety and $77 \%$ had a poor level of musical performance. The Spearman Rho hypothesis test established with a value of 0.000 that there was a significant relationship between the study variables, while the correlation coefficient of -.707 indicated that the relationship was inverse.
\end{abstract}

Keywords: music; performing arts; musical interpretation; scenic anxiety; classical music 


\section{Introducción}

La ansiedad escénica es una fobia social que se presenta ante un estímulo (examen, recital, presentación), donde el ser humano siente que pone en riesgo su autoestima e interioriza negativamente un fracaso anticipado, lo cual provoca síntomas fisiológicos (pulso acelerado, respiración descontrolada, sudoración excesiva), emocionales (nerviosismo, temor, pánico), cognitivos (falta de concentración y memorización) y conductuales (temblor en extremidades, rigidez muscular) (Kenny, 2011). Al respecto, Dalia (2004) precisa que la ansiedad escénica es definida como "una emoción que se manifiesta cuando no se debe, es comparada con un miedo ilógico a algo, a este algo se le conoce como estímulo, debido a que puede producir un efecto en nuestro organismo" (p.39).

Desde el contexto musical, Zarza-Alzugaray, Casanova-López y Robles-Rubio (2016) mencionan que existen tres factores que generan la ansiedad escénica en entornos musicales: el primero, se encarga del estudio del contexto de las relaciones tempranas del estudiante, así como las primeras experiencias de actuación; el segundo, es el encargado de evaluar la vulnerabilidad psicológica general o estado de indefensión, y el tercer factor "es el que estudia las preocupaciones concretas relacionadas con la actuación que va a acontecer" (p. 17). Para Zarza-Alzugaray, Casanova-López y Orejudo (2016), la ansiedad escénica es sufrida por músicos en conciertos con amigos o interpretando una obra musical de forma profesional. Si bien se puede afirmar que la mayoría de músicos tienen un cierto grado de ansiedad, no suelen tener problemas de forma que interfiera en su desempeño artístico. Herrera-Torres y Campoy-Barreiro (2020) mencionan que actualmente no existe una definición clara sobre la ansiedad escénica en músicos; sin embargo, el problema empieza cuando los niveles de activación psicofisiológica de la ansiedad superan el umbral soportado por estos. En ese momento es cuando sus habilidades técnicas pueden disminuir considerablemente sin probabilidad de recuperarse.

Los jóvenes estudiantes del conservatorio de música no están exentos de este problema, ya que deben someterse a exámenes en los que se les evalúa su afinación, postura, técnica musical y expresión musical; es decir, su ejecución musical, para poder acreditar y aprobar semestralmente el curso de instrumento mediante recitales o conciertos musicales. Un nivel ínfimo de nervios al momento de una presentación puede favorecer la ejecución del instrumento (Juárez, 2014); no obstante, cuando estos suben de nivel, se pueden presentar como ansiedad escénica, provocando una crisis en el estudiante, quien incide en errores durante la ejecución del instrumento o incluso llega a detener el recital. 
Algunos estudios sobre este tema han tenido diferentes hallazgos. Osborne, Kenny y Holsomback (2005) concluyeron que los niveles altos de ansiedad escénica en los estudiantes de habla inglesa se correlacionan positivamente con niveles bajos de interpretación musical. En una línea similar, Vento (2017) demostró que aquellos estudiantes que reportaron mayores puntuaciones en el nivel de ansiedad también reportaron una mayor tendencia a enfocarse en el malestar o las emociones no placenteras que sienten, lo cual suele estar relacionado con un bajo control musical. Por su parte, en el estudio de Goijman (2018), se precisa que si bien se evidenciaron ciertos niveles de ansiedad en los intérpretes musicales, no existe una relación directa entre la ansiedad y su rendimiento musical. En cambio, Herrera, Manjón y Quiles (2019) demostraron que los síntomas cognitivos prevalecen en mayor nivel que los sintomas fisiológicos durante la ejecución musical, situación que hace menester de estrategias de afrontamiento para la superación de la ansiedad. Por último, en el caso de una muestra de músicos de la fundación Simón Bolívar en la Ciudad de Maracaibo, Atencio (2015) halló que los intérpretes mostraron niveles moderados de ansiedad escénica en el momento de responder a la evaluación. En cuanto a la relación del tipo de instrumento y la ansiedad escénica, el autor encontró que esta se manifestaba en un nivel muy bajo en el canto y en un nivel moderado en los grupos de cuerdas frotadas, viento madera, viento metal, cuerda pulsada, percusión y piano.

En el caso del Conservatorio Regional de Música Luis Duncker Lavalle, se han venido reportando bajos niveles de ejecución musical en los recitales de música. Sin embargo, no se tiene respuesta sobre una posible asociación con la ansiedad escénica. Por tal razón, el presente artículo tiene como objetivo principal evaluar la relación entre este fenómeno y la ejecución musical de los estudiantes. Para ello se toma una muestra de alumnos de la Especialidad de Cuerdas Frotadas.

\section{Metodología}

La metodología utilizada en el estudio se centró en los criterios de una investigación básica, que se caracteriza por no tener fines prácticos de forma inmediata. Su objetivo principal es incrementar la teoría y principios fundamentales con respecto al fenómeno de estudio (Hernández-Sampieri \& Mendoza, 2018). El nivel de investigación fue el correlacional, el cual se fundamenta en la asociación o relación entre dos variables de estudio cualitativas o cuantitativas (Arias \& Covinos, 2021); los resultados de las varibles de estudio no se alteraron. La investigación se realizó en un solo periodo, por lo que el diseño es no 
experimental, de corte transversal. La población estuvo constituida por 48 estudiantes de cuerdas frotadas (violín, viola, violonchelo, contrabajo), de primer año a quinto año de la carrera profesional de Interpretación Musical del Conservatorio Regional de Música Luis Duncker Lavalle. Para hallar la muestra se utilizó el método no probabilístico-por conveniencia, por tal razón se eligió a la población censal, es decir, los 48 estudiantes los cuales conformaron la muestra de estudio.

Para medir la ansiedad escénica se utilizó la técnica del test, particularmente se empleó el "Test del pingüino"; este corresponde a un instrumento de 20 ítems que mide la ansiedad escénica en músicos. Dicho instrumento fue elaborado y validado por Eduardo Juárez López en su trabajo de investigación Ansiedad Escénica: la cara oculta del artista" (2014). Este se codificó mediante la escala tipo Likert, con valores asignados de: siempre (5), casi siempre (4), indiferente (3), casi nunca (2), nunca (1), y se calificó en: alto (71-100 puntos), medio (41-70) y bajo (20-40).

En cuanto a la medición de la ejecución musical, se utilizó la técnica de la observación y la rúbrica de evaluación como instrumento, el cual estuvo conformado por 10 elementos. El instrumento fue validado por juicio de expertos y por el método Alfa de Cronbach, con un valor Recuperado de .886. La codificación fue: cumple con todos los criterios (2 puntos); cumple con el $50 \%$ de los criterios (1 punto); cumple con menos del $50 \%$ de los criterios (0.5 punto). La calificación fue de: excelente (18-20 puntos), bueno (15-17 puntos), regular (12-14 puntos) y deficiente (01-11 puntos).

\section{Resultados}

La recolección de datos se realizó de forma física antes y durante la presentación del recital de música para fin de semestre durante el año 2020. Se les tomó la prueba de ansiedad escénica 10 minutos antes del recital de forma colectiva y la observación se tomó de forma individual durante el recital, según el turno de cada estudiante. 
Ansiedad escénica y ejecución musical en estudiantes del conservatorio de música en Arequipa, 2020

Tabla 1. Nivel de ansiedad escénica en estudiantes del conservatorio de música

\begin{tabular}{|c|c|c|}
\hline & Frecuencia & Porcentaje \\
\hline Alto & 34 & $71 \%$ \\
\hline Medio & 3 & $6 \%$ \\
\hline Bajo & 11 & $23 \%$ \\
\hline
\end{tabular}

Fuente: datos obtenidos del instrumento de medición.

En la tabla 1, los resultados muestran que el $71 \%$ de estudiantes tienen un nivel alto de ansiedad escénica, lo que representa en su mayoría a estudiantes de primer y segundo año de los instrumentos viola y contrabajo. Asimismo, durante el recital de música se mostraron agitados, desconcentrados y sudorosos. Aquellos que tuvieron un nivel bajo de ansiedad escénica fueron, en su mayoría, estudiantes de segundo y quinto año de los instrumentos musicales violín y violonchelo.

Tabla 2. Nivel de ejecución musical de los estudiantes del conservatorio de música

\begin{tabular}{|c|c|c|}
\hline & Frecuencia & Porcentaje \\
\hline Excelente & 2 & $4 \%$ \\
\hline Bueno & 8 & $17 \%$ \\
\hline Regular & 1 & $2 \%$ \\
\hline Deficiente & 37 & $77 \%$ \\
\hline
\end{tabular}

Fuente: datos obtenidos del instrumento de medición.

En la tabla 2, los resultados muestran que el $77 \%$ de estudiantes tienen un nivel deficiente de ejecución musical, lo que representa en su mayoría a estudiantes de primer y segundo año de los instrumentos de viola y contrabajo. Durante el recital de música estos estudiantes tuvieron bastantes desafinaciones y se perdían en el tiempo, el ritmo estuvo desacompasado y se olvidaron algunas notas del tema musical. Aquellos estudiantes que tuvieron un nivel bueno de ejecución musical fueron, mayoritariamente, estudiantes de segundo y quinto año de los instrumentos musicales violín y violonchelo. Además, fueron afinados y con sentido del ritmo en la mayor parte del tema musical interpretado. 
Tabla 3. Prueba de normalidad

\begin{tabular}{|c|c|c|c|c|c|c|}
\hline & \multicolumn{9}{|c|}{ Pruebas de normalidad $^{\text {Kolmogorov-Smirnov }}$} & \multicolumn{3}{c|}{ Shapiro-Wilk } \\
\hline Interpretación musical & Estadístico & gl & Sig. & Estadístico & gl & Sig. \\
\hline Ansiedad escénica & .258 & 8 & .000 & .752 & 6 & .000 \\
\hline a. Corrección de significación de Lilliefors \\
\hline
\end{tabular}

Fuente: IBM SPSS Stadistic 25, recuperado de la sistematización de datos.

De acuerdo con la regla:

- Si el nivel de significancia es $<0.05$ = la población no tiene una distribución normal.

- Si el nivel de significancia es > 0.05 = la población tiene una distribución normal.

El resultado obtenido de .000 permite establecer que la población no tiene una distribución normal. Por tal razón, se utiliza una prueba no paramétrica denominada Rho de Spearman.

Tabla 4. Tabla cruzada de resultados de las variables de estudio

\begin{tabular}{|c|c|c|c|c|c|c|c|}
\hline & & & \multicolumn{5}{|c|}{ Ejecución musical } \\
\hline & & & Deficiente & Regular & Bueno & Excelente & Total \\
\hline \multirow{6}{*}{$\begin{array}{l}\text { Ansiedad } \\
\text { escénica }\end{array}$} & \multirow{2}{*}{ Bajo } & Recuento & 2 & 0 & 7 & 2 & 11 \\
\hline & & $\%$ del total & $4 \%$ & $0 \%$ & $15 \%$ & $4 \%$ & $23 \%$ \\
\hline & \multirow{2}{*}{ Medio } & Recuento & 3 & 0 & 0 & 0 & 3 \\
\hline & & $\%$ del total & $6 \%$ & $0 \%$ & $0 \%$ & $0 \%$ & $6 \%$ \\
\hline & \multirow{2}{*}{ Alto } & Recuento & 32 & 1 & 1 & 0 & 34 \\
\hline & & $\%$ del total & $67 \%$ & $2 \%$ & $2 \%$ & $0 \%$ & $71 \%$ \\
\hline \multirow{2}{*}{\multicolumn{2}{|c|}{ Total }} & Recuento & 37 & 1 & 8 & 2 & 48 \\
\hline & & $\%$ del total & $77 \%$ & $2 \%$ & $17 \%$ & $4 \%$ & $100 \%$ \\
\hline
\end{tabular}

Fuente: datos obtenidos de la sistematización de los resultados en IBM SPSS 25 stadistic. 
Ansiedad escénica y ejecución musical en estudiantes del

Artículo conservatorio de música en Arequipa, 2020

La tabla 4 muestra los resultados de las variables "ansiedad escénica" y "ejecución musical", los cuales evidencian que el $67 \%$ estudiantes con un nivel alto de ansiedad escénica también tuvieron una ejecución musical deficiente. Los resultados demuestran que existe una correlación inversamente proporcional entre las variables de estudio.

\section{Prueba de hipótesis}

Previamente, para definir la prueba de hipótesis, se realizó la prueba de normalidad para establecer la distribución de la población. Se obtuvo como resultado un nivel de significancia de .000 (Parámetros de Shapiro-Wilk), lo cual indica que los datos no tuvieron una distribución normal. De igual manera, al tratarse de variables cualitativas con escala ordinal, se determina la prueba estadística Rho de Spearman para la prueba de hipótesis:

- H1: existe relación significativa e inversa entre la ansiedad escénica y la ejecución musical de los estudiantes de la Especialidad de Cuerdas Frotadas del Conservatorio Regional de Música Luis Duncker Lavalle.

- Hø: no existe relación significativa e inversa entre la ansiedad escénica y la ejecución musical de los estudiantes de la Especialidad de Cuerdas Frotadas del Conservatorio Regional de Música Luis Duncker Lavalle.

Tabla 5. Prueba Rho de Spearman para las variables de estudio

\begin{tabular}{|c|c|c|c|c|}
\hline & & & Ansiedad escénica & Ejecución musical \\
\hline \multirow{6}{*}{$\begin{array}{c}\text { Rho } \\
\text { de Spearman }\end{array}$} & \multirow{3}{*}{$\begin{array}{l}\text { Ansiedad } \\
\text { escénica }\end{array}$} & $\begin{array}{l}\text { Coeficiente } \\
\text { de correlación }\end{array}$ & 1.000 & $-.707^{* *}$ \\
\hline & & Sig. (bilateral) & & 0.000 \\
\hline & & $\mathrm{N}$ & 48 & 48 \\
\hline & \multirow{3}{*}{$\begin{array}{l}\text { Ejecución } \\
\text { musical }\end{array}$} & $\begin{array}{c}\text { Coeficiente } \\
\text { de correlación }\end{array}$ & $-.707^{\star *}$ & 1.000 \\
\hline & & Sig. (bilateral) & 0.000 & \\
\hline & & $\mathrm{N}$ & 48 & 48 \\
\hline
\end{tabular}

Fuente: datos obtenidos de la sistematización de los resultados en IBM SPSS 25 stadistic.

ESCENA. Revista de las artes, 2021, Vol. 81, Núm. 1 (julio-diciembre), pp. 170-182 
En la tabla 5, se muestran los resultados para la prueba de hipótesis Rho de Spearman entre las variables de estudio: "ansiedad escénica" y "ejecución musical". El valor de significancia obtenido es de 0.000 con lo que se establece el rechazo de la hipótesis nula: no existe relación significativa e inversa entre la ansiedad escénica y la ejecución musical de los estudiantes de la Especialidad de Cuerdas Frotadas del Conservatorio Regional de Música Luis Duncker Lavalle. De esta forma, se acepta la hipótesis de investigación: existe relación significativa e inversa entre la ansiedad escénica y la ejecución musical de los estudiantes de la Especialidad de Cuerdas Frotadas del Conservatorio Regional de Música Luis Duncker Lavalle. Además, el coeficiente de correlación de -.707 indica que la relación entre las variables de estudio es inversa y significativa.

\section{Discusión de resultados y conclusiones}

Antes de discutir los resultados, es importante mencionar que una de las limitaciones de estudio fue el tamaño de la población, dado que el Conservatorio Regional de Música Luis Duncker Lavalle cuenta con un pequeño universo de estudiantes de distintos instrumentos, de los cuales solo 48 estudian cuerdas frotadas (27 violines, 13 violonchelos, 4 violas y 4 contrabajos). No se incluyó a estudiantes de otros instrumentos musicales debido a que los criterios de evaluación son diferentes a los de cuerdas frotadas en cuanto a afinación, postura y vibrato (este último aspecto es característico de los instrumentos de cuerda frotada). Incluso, en otros instrumentos no es necesario evaluar la afinación o el vibrato.

Los resultados demostraron que la mayoría de estudiantes tuvieron un nivel alto de ansiedad escénica y un nivel deficiente de ejecución musical. También, se evidenció una relación inversa y significativa entre ambas variables de estudio. Estos resultados son similares a los presentados por Vento (2017), quien, como se mencionó anteriormente, concluyó que los estudiantes con altos niveles de ansiedad presentaron también falta de control musical.

En cuanto a los niveles de ejecución musical evaluados, por una parte, es relevante tener en cuenta que en aquellos estudiantes con niveles deficientes, pueden haber intervenido factores como la falta de estudio, de práctica o de lectura del instrumento. Por otra parte, Zarza-Alzugaray, Casanova-López y Robles-Rubio (2016) mencionan otra serie de factores que influyen en niveles altos de ansiedad escénica, como la relación del estudiante con el público, su estado de indefensión y la percepción sobre el presente y futuro (durante y despues del concierto), los cuales también pueden influir en un desempeño deficiente. 
Ansiedad escénica y ejecución musical en estudiantes del

Artículo conservatorio de música en Arequipa, 2020

En relación con lo anterior, en el Conservatorio Regional de Música Luis Duncker Lavalle, no existe un programa, curso o taller que permita controlar y disminuir los niveles de ansiedad, lo que consecuentemente se representa en los altos niveles de ansiedad encontrados. Estos resultados negativos son un problema ahora y pueden extenderse en un futuro si no se interviene. Como señalan Zarza-Alzugaray, Casanova-López y Orejudo (2016), además de las consecuencias ya mencionadas, los estudiantes pueden presentar dificultades conductuales como evitación y escape. Si estos síntomas se agravan, será necesario un tratamiento que iría más allá de la realización de espacios formativos para el manejo de este tipo de ansiedad. 


\section{Referencias}

Arias, J. L. \& Covinos (2021). Diseño y metodología de la investigación. Perú: Ediciones Enfoques Consulting.

Atencio, E. (2015). Ansiedad Escénica en Músicos de la Fundación Musical Simón Bolivar en la Ciudad de Maracaibo (tesis de pregrado). Universidad Rafael Urdaneta, Maracaibo, Venezuela.

Dalia, G. (2004). Cómo superar la ansiedad escénica en músicos. Madrid: Mundimúsica.

Goijman, J. J. (2018). Niveles de ansiedad y percepción de rendimiento en intérpretes musicales. Investigaciones en psicología, 23(1), 37-43. Recuperado de http://www.psi. uba.ar/investigaciones/revistas/investigaciones/indice/trabajos_completos/anio23_1/ goijman.pdf

Hernández-Sampieri, R. \& Mendoza, C. (2018). Metodología de la investigación. México: Mc Graw Hill.

Herrera, L., Manjón, J., y Quiles, O. L. (2019). Ansiedad escénica en alumnos de flauta traversa de conservatorio. Mexicana de Psicología, 32(2), 169-181. Obtenido de https:// www.redalyc.org/pdf/2430/243045364007.pdf

Herrera-Torres, L. \& Campoy-Barreiro, C. (2020). Ansiedad escénica musical en profesorado de conservatorio: frecuencia y análisis por género. Revista de Psicología y Educación, 15(1), 32-47. doi: 10.23923/rpye2020.01.184

Juárez, E. (2014). Ansiedad Escénica: la cara oculta del artista (tesis de maestría). Centro de Estudios e Investigación Guestálticos, Xalapa, México.

Kenny, D. (2011). The Psychology of Music Performance Anxiety. Australia: Oxford.

Osborne, M.S., \& Kenny, D.T. (2005). Development and validation of a music performance anxiety inventory for gifted adolescent musicians. Journal of Anxiety Disorders, 19(7), 725-751. doi:10.1016/j. janxdis.2004.09.002

Vento, R. M. (2017). Ansiedad y afrontamiento en estudiantes de un conservatorio de música. Lima: Pontificia Universidad Católica del Perú. 
Ansiedad escénica y ejecución musical en estudiantes del

Artículo conservatorio de música en Arequipa, 2020

Zarza-Alzugaray, F. J., Casanova-López O., \& Orejudo, S. (2016). Estudios de música en los conservatorios superiores y ansiedad escénica en España. Revista Electrónica Complutense de Investigación en Educación Musical, 13, 50-63. Recuperado de https:// revistas.ucm.es/index.php/RECl/article/view/49442/50113

Zarza-Alzugaray, F. J., Casanova-López, O., \& Robles-Rubio, J. E. (2016). Relación entre la ansiedad escénica, perfeccionismo y calificaciones en estudiantes del título Superior de Música. Reidocrea, 5, 16-21. Recuperado de http://www.ugr.es/ reidocrea/5-3.pdf 